\title{
Ecodesarrollo como estrategia para la sostenibilidad: un análisis socioambiental basado en la obra de Ignacy Sachs
}

\section{Ecodesenvolvimento como estratégia para a sustentabilidade: uma análise socioambiental baseada na obra do Ignacy Sachs}

Ecodevelopment as a strategy for sustainability: a socioenvironmental analysis in the work of Ignacy Sachs

Luciana Virginia Mario Bernardo ${ }^{1}$

Cesar Benites Mario Zidora ${ }^{2}$

Maycon Jorge Ulisses Saraiva Farinha ${ }^{3}$

Nelson David Lesmo Duarte ${ }^{4}$

${ }^{1}$ Doctoranda en Desarrollo Regional y Agronegocios por la Universidad Estadual del Oeste de Paraná (Unioeste). Maestría en Agronegocios y Licenciatura en Ciencias Contables, por la Universidad Federal de la Grande Dourados (UFGD).

E-mail: lucianamario@yahoo.com.br

2 Doctorando en Desarrollo Regional y Agronegocios por la Universidad Estadual del Oeste de Paraná (Unioeste). Maestría en Agronegocios por la Universidad Federal de Goiás y Agrónomo Universidad Eduardo Mondlane, Moçambique.

E-mail: cesarbmzidora@hotmail.com

${ }^{3}$ Maestría en Agronegocios y Geógrafo, ambos por la Universidad Federal de la Grande Dourados (UFGD).E-mail: maycondds@hotmail.com

${ }^{4}$ Maestría en Agronegocios, por la Universidad Federal de la Grande Dourados (UFGD), y administrador por la Universidad Nacional de Asunción Facultad de Ciencias Agrarias. E-mail: nelson.david@hotmail.com 
Luciana Virginia Mario BERNARDO; Cesar Benites Mario ZIDORA; Maycon Jorge Ulisses Saraiva FARINHA; Nelson David Lesmo DUARTE

Resumen: El objetivo de abordar el ecodesarrollo propuesto por Ignacy Sachs en sus obras literarias y mostrar la producción académica y científica de Brasil, asociadas al ecodesarrollo propuesto y discutido por Sachs. En términos metodológicos, la investigación de carácter bibliográfico. Los resultados apuntan que, para concretar el ecodesarrollo en todas las dimensiones, será necesario una nueva postura en términos de padrón de producción y de consumo, a partir del uso de tecnologías limpias en todo proceso de producción. Se apunta que la pobreza y el estilo de producción capitalista como los principales factores que más daños causan al ambiente, resultando en alteración del equilibrio ecológico y de todo el ecosistema, poniendo en riesgo el bienestar social de las futuras generaciones.

Palabras clave: equilibrio ecológico; medio ambiente; desarrollo endógeno.

Resumo: O artigo tem como objetivo abordar o ecodesenvolvimento proposto por Ignancy Sachs em suas obras literárias e mostrar a produção acadêmica e científica do Brasil, associada ao ecodesenvolvimento proposto e discutido por Sachs. Em termos metodológicos, a pesquisa foi bibliográfica, descritiva e qualitativa. Os resultados da pesquisa apontam que, para se efetivar o ecodesenvolvimento ou desenvolvimento sustentável em todas as suas dimensões, é necessária uma nova postura em termos de padrões de produção e de consumo, a partir do uso tecnologias limpas em todo processo extrativo, produtivo e de conversão dos recursos em bens e serviços. E, ainda, adoção de formas de energias renováveis que menos danos causam ao ambiente para garantir a satisfação das necessidades do homem, uma vez que estas são ilimitadas e os seus recursos limitados. Apontou-se a pobreza e o estilo de produção capitalista como os principais fatores que mais danos causam ao ambiente, resultando em alteração do equilíbrio ecológico e de todo ecossistema, pondo em risco o modo de vida, primeiro, da geração atual e, em seguida, o bem-estar social das futuras gerações.

Palavras-chave: meio ambiente; desenvolvimento endógeno; equilíbrio ecológico.

\begin{abstract}
Ignacy Sachs in his literary works and show the academic and scientific production of Brazil, associated with ecodevelopment proposed by Sachs. In methodological terms, the research was bibliographical. The results point out that, in order to achieve ecodevelopment in all its dimensions, a new posture is required in terms of production and consumption patterns, using clean technologies in the production process. Poverty and the capitalist production style were pointed out as the main factors that cause the most damage to the environment, resulting in a change in the ecological balance and in the whole ecosystem, putting at risk the social well-being of future generations.
\end{abstract}

Keywords: ecological balance; environment; endogenous development. 


\section{INTRODUCCIÓN}

Los problemas ambientales se tornaron evidentes desde la década de 1960 y, en las décadas siguientes, la cuestión pasa por la amenaza que se tornó visible para las personas a partir de catástrofes ambientales. Por otro lado, la conferencia de Estocolmo sobre el medio ambiente, ocurrida en 1972, es considerada el marco histórico-político internacional sobre los problemas ambientales. A través de ella proporcionó que las discusiones globales sobre el medio ambiente fueran iniciadas y sean convertidas en políticas públicas. El enfoque discutido en la conferencia por los miembros participantes de diversas naciones fue la gestión de recursos ambientales. El aspecto adoptado se refiere a las generaciones futuras y sus derechos a la biodiversidad existente en el mundo. De esta forma, el uso de los recursos ambientales en el tiempo presente, debe partir de la conjetura de la sostenibilidad para estar disponible en el futuro.

Las preocupaciones con el medio ambiente que surgieron en los años 1970 proporcionaron entendimiento nuevo para que el ecodesarrollo fuera propuesto con una alternativa para el desarrollo de los países. La forma de desarrollo pasó a ser denominada "Desarrollo Sostenible", después del Informe de Brundtland. En esta forma de comprender el desarrollo existe un énfasis en la viabilidad económica de las actividades proporcionales a la importancia de la solidaridad humana con los aspectos sociales y ambientales correspondientes a la generación presente y futura.

En la perspectiva de la economía ecológica que Sachs se conceptuó el ecodesarrollo. En este concepto no son realizadas importaciones de modelos, más se desarrolla práctica a partir de las características de los ecosistemas locales, de la cultura y las características ambientales. La importancia del ecodesarrollo se relaciona a un conjunto de factores, siendo ellos: la percepción holística sobre el desarrollo, el bienestar social y sus relaciones con la generación futura a partir de la solidaridad de los habitantes del momento actual con aquellos que todavía no nacieron. Se desprende, así, que la satisfacción de las necesidades humanas está proyectada para diferentes periodos, considerando el largo plazo.

El objetivo del presente artículo es, por lo tanto, abordar el ecodesarrollo propuesto por Ignacy Sachs en sus obras literarias y mostrar 
la producción académica y científica de Brasil, asociadas al ecodesarrollo propuesto y discutido por Sachs. La investigación no pretende concluir la temática de la sostenibilidad, pues el tema es amplio y viene siendo discutido por diversos autores. Diferentemente, el objetivo es hacer un análisis del enfoque de Sachs sobre el asunto con el recorrer de los años de su investigación, indicando las alteraciones en las dimensiones de la sostenibilidad. La metodología adoptada es bibliométrica descriptiva y cualitativa.

\section{ENFOQUE TEÓRICO DE LOS AVANCES DEL CONCEPTO DE ECODESARROLLO}

En 1970, Paul Ehrlich hablaba sobre la importancia de los países subdesarrollados direccionaren sus recursos para el desarrollo de la producción de alimentos con enfoque ecológico, reduciendo sus empeños para el alcance de la industrialización (MCCORMICK, 1992). A partir de ese dialogo, en 1973 fue utilizado el vocabulario ecodesarrollo por Maurice Strong, eso durante la primera reunión del Consejo Administrativo de Ginebra (RAYNAUT; ZANONI, 1993). La intención era definir un estilo de desarrollo para las áreas rurales localizadas en países subdesarrolladas, a partir de dos criterios: (i) recursos locales y (ii) conocimientos tradicionales de los residentes rurales. De esta forma, la nueva propuesta visaba satisfacer dos necesidades, que sean o no la destrucción de los recursos naturales y la manutención de la biodiversidad para la generación futura. Se confía en la posibilidad de este desarrollo debido a la ausencia de acciones depredadoras por parte de los pueblos localizados en estos países. Además de eso, se propone una alternativa de desarrollo a partir de las características locales para que no haya importaciones de modelos extranjeros (SACHS, 1980).

Las características del concepto de ecodesarrollo fueron, con todo, determinadas solamente en 1974, cuando Sachs retomó el vocablo y lo divulgo en esfera global (ARAÚJO; BARROSO; SOUZA, 2014). Para Sachs (1986), los principios determinados en aquel momento fueron: (i) satisfacción de las necesidades básicas; (ii) solidaridad con las generaciones futuras; (iii) participación de la población; (iv) preservación del medio ambiente; (v) existencia de un sistema de garantía de empleo, de seguridad social y de 
respeto cultural y (vi) programas culturales. De esta forma, Montibeller Filho (2004) considera el ecodesarrollo como una forma de desarrollo que es realizado a partir de las potencialidades de las ecorregiones, reduciendo dependencias externas, respetando el medio ambiente y manteniendo recursos para las futuras generaciones.

En el contexto de la época, el ecodesarrollo representó una nueva forma de desarrollo, una innovación para la bipolarización existente (ANDRADE, 2008; FERNANDEZ, 2011). Las vías consolidadas se referías al "crecimiento cero" insertada en el Informe de Meadows publicado en el Club de Roma, en el cual creía que la limitación del crecimiento económico estaba en la escasez de los recursos naturales y en la polución (MEADOWS et al., 1972). El primer posicionamiento fue caracterizado como "Neomalthusiano". Y la segunda vía relataba las exigencias de los países subdesarrollados en crecer del mismo modo como aquellos considerados desarrollados. En la perspectiva de los desarrollistas y de los "cornocupianos", o sea, los entusiastas de las tecnologías, el conjunto envolviendo trabajo, capital y tecnología sería suficiente para solucionar los problemas causados por la degradación ambiental (SOLOW, 1974).

Se denota que la cuestión central no es escoger entre desarrollar o preservar el medio ambiente, la opción por la manera de desarrollar contemplando o no el medio ambiente (SOUTH COMISSION, 1990). Se cree que las poblaciones presentes en países subdesarrollados no pueden dejar de desarrollarse, pero que pueden optar por la forma de como el desarrollo será realizado. Se observó que, en esa perspectiva, se denota la importancia del desarrollo rural, con todo eso no es el único responsable para determinar el desarrollo que opte por la interacción con el medio ambiente. Se debe dar atención también a los espacios urbanos, pues en ellos hay tendencia de crecimiento poblacional al transcurrir de los años, de forma a concretar los consumidores en esos locales (SACHS, 1993).

Sachs (1986) considera que el ecodesarrollo es endógeno, pues promueve soluciones específicas para los problemas de los ecosistemas. Se debe pautar en las dinámicas ecológica y cultural, buscando obtener repuestas para las necesidades inmediatas, como también para aquellas que constituyen el largo plazo. Esa forma de desarrollar desconsidera la 
cuestión excesiva de ayuda externa, priorizando la interna, esto es, dando más voto de confianza a la capacidad de las sociedades humanas de identificar sus problemas y de crear soluciones originales, mismo teniendo como inspiración la réplica de experiencias de los otros.

El Ecodesarrollo surge a partir de una polémica dupla contra los partidarios del crecimiento salvaje, que defendían una fuga para frente al mal desarrollo, como medio para corregir los males y contra los zeritas, víctima de absolutismo del criterio ecológico a punto de perder la visión antropocéntrica de modo, que es la de todas las filosofías humanistas. (SACHS, 1986, p. 113).

Esa forma de desarrollo tiene su base conceptual intrínsecamente ligada a la gestión de los recursos naturales. Ese hecho implica una forma difícil de convencimiento social contra los desperdicios de los recursos naturales y exige medidas de alteraciones de las estructuras de consumo, de la durabilidad de los productos, entre otros factores (SACHS, 1986).

El gran motivo que está en el origen de los problemas ecológicos es la mala distribución de los recursos, esto es, existencia de la desigualdad social que engendra desperdicios de recursos en los ricos y la súper exploración de la tierra por parte de los pobres. Cuanto la polución, ella es fruto de la abundancia, mas también de la miseria, de las fábricas y de los automóviles, mas también de los barrancos sin agua potable ni desagüe. De ahí que será posible haber soluciones durables a los problemas ecológicos de nuestro planeta que no sea las fundamentadas en la igualdad. Principalmente porque la igualdad constituye condiciones previas de toda discusión seria acerca de la limitación voluntaria del volumen de consumos de los materiales, cuestión que demostramos en nuestro conocimiento. (SACHS, 1986, p. 45).

Además de eso, Sachs (1986) considera que el ecodesarrollo necesita ser operacional a partir de estrategias de acciones que promuevan soluciones a través de equilibrio ecológico y de la calidad de recursos naturales. Ambas características son utilizadoras por la tecnología para construir bienes y servicios consumibles por la sociedad. Así, la operacionalizad será obtenida a partir de tres condiciones (i) conocimiento cultural y sobre el ecosistema, (ii) planeamiento del desarrollo, con participación popular y (iii) dominio institucional, con el mínimo la existencia de mercados que promuevan acceso a los diferentes individuos. De esa forma, el desafío de este desarrollo 
se refiere a la identificación y la satisfacción de las necesidades de cada persona y de toda la población, en base sostenible, respetando su diversidad y su potencialidad creativa de cambios.

Inicialmente fueron consideradas cinco dimensiones de sostenibilidad en ese formato de desarrollo: (i) social, (ii) económica, (iii) ecológica, (iv) espacial y (v) cultural. La dimensión "i" tiene objetivo la existencia de mayor equidad en la sociedad, de forma que el patrón de vida de los individuos sea aproximado entre ellos. La dimensión "ii" implica mejor asignación de los recursos existentes en el país, de forma a reducir las externalidades negativas inherentes a la retirada de recursos financieros para otros países por medio de dividas. La dimensión "iii" se refiere al uso de medidas capaces de la promoción de la conservación de la biodiversidad de los ecosistemas, contemplando mejor uso de los recursos naturales, reducción de residuos, innovación tecnológica de bajo impacto ambiental y conservación ambiental. La dimensión "iv" remite a la distribución territorial, de forma a crear equilibrio entre el asentamiento de las personas en el espacios rurales y urbanos. Y, a final, la dimensión " $\mathrm{V}$ " trae la incorporación de las características culturales locales para el desarrollo (SACHS, 1993).

Se percibe que Sachs opta por el uso de termino ecodesarrollo, mismo que este haya pasado a ser denominado Desarrollo Sostenible en el Informe Brundtland. Después, con todo, en 2002, se vuelta a revisar y presentar ocho dimensiones, ampliando el concepto: (i) social, (ii) cultural, (iii) ecológica, (iv) ambiental, (v) territorial, (vi) económica, (vii) política nacional, y (viii) política internacional. Así el promueve la alteración de la dimensión espacial para territorial e inclusión de las dimensiones ambiental, política nacional y política internacional. La dimensión territorial se remite al equilibrio entre el urbano y el rural a partir del desarrollo de las regiones, atribuyendo a la mejor calidad al urbano. La dimensión ambiental se refiere a respeto al ecosistema natural y a su capacidad de restaurar las características naturales. En relación a la política nacional, resalta la importancia de la democracia y de la interacción entre el gobierno y los emprendedores para establecer convenio en la realización de proyectos. Y la política internacional tangencia a la cooperación entre los países para tratar de los asuntos económicos, sociales y ambientales (SACHS, 2002). 
Para Salamoni y Gerardi (2001), la propuesta listada para el ecodesarrollo es una forma de auxilio para que la sociedad sea educada para valorizar las características ambientales del ecosistema natural en que está localizada. A partir de esta forma de desarrollo, se adicionan aspectos cualitativos al tema, que, además del conocimiento económico, aborda costos ambientales y sociales. De todo eso, el objetivo es conciliar el grado de satisfacción de la población con la limitación de los recursos naturales.

\section{ANÁLISIS DE LA RELACIÓN ENTRE EL ECODESARROLLO Y LA PRESERVACIÓN AMBIENTAL}

La teoría del ecodesarrollo relaciona la ecorregión natural con la población, con la técnica y con los recursos naturales utilizados para la producción. La relación propuesta busca, de la mejor manera una posible, integración de los componentes de forma sustentable a largo plazo. A principio se cree que, por medio del uso de técnicas específicas, es posible transformar los recursos en productos para fines de consumo y de reproducción social, y, simultáneamente, esas mismas técnicas deben ser limpias, no causando daños al medio ambiente (SACHS, 1986).

Para Sachs (1986) y para el Consejo Mundial sobre el Medio Ambiente y Desarrollo (CMMAD, 1988), la pobreza es unas de las principales causas de la problemática ambiental, pues culmina con la degradación del medio ambiente. Siendo así, es de la responsabilidad de los gobiernos y de las instituciones el papel regulador de la protección y de la preservación ambiental y de crear posibilidad de separar las cuestiones que articulan al respecto al desarrollo económico de las cuestiones relativas al medio ambiente, eso porque la manera como las sociedades se desarrollan reduce la biodiversidad de los recursos ambientales y, en algunos casos, eso resulta en la escasez de determinado recurso.

En relación a los países subdesarrollados, la CMMAD (1988, p. 30) resalta:

El desgaste del medio ambiente fue con frecuencia el resultado de la creciente demanda de recursos escasos y la polución causada por la mejoría de patrón de vida de los relativamente ricos. Pero la propia pobreza degrada el medio ambiente, creando otro tipo de desgaste 
ambiental. Para sobrevivir, los pobres y los hambrientos muchas veces en su propio ambiente; derriban bosques, permiten pastoreo excesivo, utilizan tierras marginadas y acuden en número cada vez mayor para las ciudades ya congestionada. El efecto acumulativo de este cambio llega al punto de hacer la propia pobreza uno de los mayores flagelos del mundo.

De esta forma, el ambiente es percibido como un espacio de sobrevivencia y de perpetuación de la vida. La opción de manejo utilizada para la realizar la gestión de los recursos insertados en este espacio es fundamental para que el ambiente permanezca con esa característica. Además de eso, Sachs (2010) resalta la importancia del uso de energía renovable como la solución para la crisis energética que podrá ocurrir en el futuro.

El futuro de la agroenergía, por tanto, en gran parte, de nuestra capacidad de transformar en biocombustible las pajas, hojas y otros desechos agrícolas y forestales, en transformar los residuos en riquezas, como proclamaba antiguamente un Slogan maoísta de consonancia bien capitalista. La parte de la producción primaria liquida de biomasa que la humanidad consume o destruye todo el año se elevará, según ciertos autores, la $40 \%$, y hasta $50 \%$ del total disponible. Calculamos peligrosamente los limites últimos: solo podremos contar con una única duplicación del consumo (y/o de destrucción), al paso que durante el próximo medio siglo la población mundial aumentaría 50\%. (SACHS, 2010, p. 29-32).

Sachs (1986) muestra que todavía las herramientas tradicionales de los economistas, sobre todo, las ofrecidas por la microeconomía, se muestran claramente insuficientes en el enfoque de la armonía del desarrollo socioeconómico con la gestión racional de los recursos y del ambiente. La regla del funcionamiento de la economía capitalista es la internacionalización del lucro por la empresa y la externacionalización, siempre posible, de los costos, que, posteriormente, van a reflejar negativamente en la sociedad (costos sociales). Siendo así, él recomienda la adopción de una nueva mentalidad de preservación y de sostenibilidad ambiental basado en la armonía y en la búsqueda de equilibrio entre economía y ecología, pues esas dos ciencias deben ser trabajadas de una forma integrada, focalizando la prosperidad de las naciones rumbo al desarrollo, respetando el capital de 
la naturaleza y evitando el máximo posible a su perturbación o destrucción.

Así, como forma de preservar el ambiente, el autor recomienda a las naciones y a la sociedad al abandono de las actuales prácticas de transformación de tierras agrícolas, en construcción urbanas y lotes de estacionamientos, o de instalaciones de industrias pesadas en áreas costeras apropiadas a acuicultura, como una necesidad de armonizar la preocupación del equilibrio ecológico y climático global y hacer que se presente en el pensamiento del desarrollo porque poco se sabe sobre las consecuencias climáticas en futuro próximo y que los posibles impactos sobre las generaciones venideras. (SACHS, 1986, p. 41).

El autor atribuye al sistema político la responsabilidad de asegurar seguridad y participación efectiva de los ciudadanos en la toma de decisiones y por proceso más democráticas en la toma de decisiones en ámbito internacional. En una visión intelectual en la búsqueda por la mayor distribución de renta y reducción de desigualdad y en el aumento de beneficios sociales, resultante de la actividad del hombre sobre la exploración y extracción de los recursos naturales, también relaciona a una condición ideal rumbo a la sostenibilidad (SACHS, 1986; 2010).

Debido al espíritu demostrado por la empresa en internalización de los lucros y en la externalización de los costos, Sachs (1986) intenta revertir esa situación, buscando más a la internalización de los beneficios sociales, en la mejoría de la calidad de vida y bienestar social de las comunidades. Esa mejoría de la calidad de vida tendría, como resultado en el ámbito social, un trabajo decentemente remunerado, llevando en cuenta el nivel de desarrollo del país, que se hace en condiciones de salubridad aceptable y que pueda dar lugar a relaciones humanas que respetan la dignidad del trabajador.

Es inconcebible reducir el desarrollo apenas al crecimiento económico. Debemos usar dos ópticas simultaneas: de un lado, el enfoque por el crecimiento, que nos remite al crecimiento del PIB, la acumulación de capital, al progreso técnico, la productividad creciente del capital, $y$, de otro enfoque que utiliza como puerta de entrada el empleo y el auto-empleo, y observa en qué medida el progreso de desarrollo favorece la inserción social por el trabajo decente de todos los que sienten la necesidad y el deseo de trabajar. (SACHS, 1986, p. 33).

Sachs (1986) crítica los padrones de uso de recursos sobre dominio del mercado y que están completamente orientados para la maximización 
de los lucros a corto plazo, siendo no sostenible a largo plazo. El autor, a través de sus enfoques teóricas y prácticas, intenta evitar que sea repetido el modelo de desarrollo usado en el enriquecimiento y en la industrialización de los países desarrollados del Norte, por ser destructivo al ambiente y no sostenible sobre el punto de vista socioambiental. $Y$ además de esta evidencia destructiva de los padrones, apunta como consecuencia los frecuentes cambios climáticos, porque ella demandara muchos recursos y muchas energías, escaseando los recursos y la aceleración de la degradación del medio ambiente, y que podrá está para acarrear alto costos sociales impuestos por la minoría en detrimento del bienestar de la mayoría.

\section{ECODESARROLLO EN EL BRASIL}

Para Oliveira y Monteiro (2015), el primer proyecto de Ecodesarrollo brasileño fue iniciado en 1978, en el estado de Minas Gerais. No fue con éxito, no obstante proporcionó a Sachs participar de otras experiencias exitosas. Como resultado, Sachs percibió que la falta de conocimiento sobre ecología cultural de la localidad impedía las discusiones sobre desarrollo. Además de eso, el país estaría embriagado por algunas características negativas del periodo colonial y que perpetuarían prácticas como clientelismo sea influyente sobre las acciones políticas. El país posee, con todo, atributos para desarrollar los aspectos sociales y ambientales. El interés de Sachs por el Brasil, en relación a sus investigaciones se constituye de diferentes factores. En este conjunto esta insertada la particularidad de haber vivido con la familia en el país, de tener constituido amistades y de encantarse por el modo de vida singular del pueblo brasileño. Además de eso, el "[...] Brasil representa un caso típico de mal desarrollo, mas consternador en la medida en que posee triunfos poderosos para levantarse (SACHS, 2009, p. 292).

La propuesta del ecodesarrollo, para Sachs (1986), incluye la toma de decisión para solucionar problemas propios de cada ecorregión. De esta forma, la ecorregión es delimitada como un espacio dotado de características específicas, espacios en el cual las soluciones de sus problemas deben ser generadas a partir de las particularidades ambientales y culturales. Deben ser contempladas las necesidades de corto y largo plazo en este proceso 
decisivo. El individuo que hace parte de este contexto y que tornará esas decisiones necesita estar consciente de su relación con la naturaleza. Caso que el hombre comprenda esa relación, entonces esta dependencia de los recursos puede ser atendida por el medio ambiental en la actualidad y también en el futuro.

Al dar énfasis al desarrollo rural, Sachs considera que ese desarrollo, para cumplir con sus finalidades de inclusión social y armonía con el medio ambiente, necesita pautarse en soluciones para la creación de conocimiento, disponibilidad de mano de obra, además de reducción de recursos económicos y naturales. Con eso, se debe utilizar la tierra a partir de innovaciones en el manejo de las culturas alimentarias de forma a obtener mayores rendimientos para el productor. No obstante, se debe limitar al uso de la tierra, tomando como parámetro las características biológicas existentes en los biomas y posibilitando que los pequeños productores tengan acceso a las innovaciones destinadas a los agronegocios. El Brasil es el espacio territorial en que esta insertada el mayor yacimiento de empleos y autoempleos contenidos en el espacio rural. Ellos auxilian a crear un ciclo de desarrollo específico para ese espacio (SACHS, 2010).

Sachs (1990) resalta la importancia del uso de los resultados agrícolas, en Brasil, como insumos para el abastecimiento de la propia cadena productiva en que es generado, como el caso de la biomasa de caña de azúcar para generación de energía para la agroindustria de la caña de azúcar. O también, para ser insumo de otra cadena productiva, como el residuo de la naranja utilizada en la producción de proteína animal. Todavía, en relación al país, considera que diferentes componentes ambientales proporcionan que la agricultura sea exitosa a partir del uso de los servicios ambientales oriundos de la biodiversidad existente. La actividad económica puede ser todavía más competitiva en mercados internacionales, a partir de prácticas de sistemas de producción agrícola, como la agroforestal y acuicultura.

El ecodesarrollo puede ser pensado a partir de la biodiversidad de los biomas existentes en Brasil y de la inversión en una nueva revolución agropecuaria que está pautada en el uso del conocimiento, en la permanencia de la población en el espacio rural y en reducción del uso de capital y en recursos naturales. Se apunta, sin embargo, que, mismo siendo destaque 
en la producción alimentaria, Brasil consiguió tres resultados a largo plazo de su historia: (i) tener una agricultura moderna, con reducción en el número de trabajadores; (ii) colonizar la Amazonia de forma socialmente frágil y ambientalmente depredador y (iii) excluir las personas del espacio rural, que colocaron en una urbanización prematura y excluyente. En los diferentes espacios rural y del urbano, hay una sociedad brasilera en extrema desigualdad (SACHS, 2001).

Al dar énfasis la cuestión de la Amazonia, Sachs (2009) considera que la mirada direccionada a esa región del país debe transbordar la conservación ambiental e inserir en el contexto las 20 millones de personas que residen en ese espacio. Muchas de esas personas viven en condiciones precarias de sobrevivencia. Se trata de persona insertada en áreas urbanas, lo que posibilita al autor identificar la Amazonia como un bosque urbanizada, un bosque que, dentro de sus especies de flora y fauna amenazadas, están también personas que allí viven. La conservación y el desarrollo de la Amazonia solo serán posibles cuando el Brasil piensa a partir de sus características.

De forma general, considerando la posibilidad de virtuosidad ambiental y social en el Brasil, resalta la infelicidad de las decisiones tomadas en el país, decisiones que generan masacres en las características socioambientales debido al manejo adoptado en las prácticas agropecuaria y en el tratamiento dado a la población. Mismo así, sin embargo, todavía se tiene millones de hectáreas en la Amazonia y reservas de tierras agrícolas todavía no exploradas y pastos extensivos que pueden ser convertidos en cultivos. Esas áreas pueden adoptar medidas de ecodesarrollo para ser utilizadas (SACHS, 2010).

\section{PRODUCCIÓN ACADÉMICA NACIONAL UTILIZANDO EL CONCEPTO DE ECODESARROLLO}

Prácticas de análisis bibliométrica retratan la intención de indicar el estado de la parte sobre determinado tema de investigación. En este presente caso, lo que quiere es apuntar al uso de concepto de ecodesarrollo en las investigaciones relacionadas al Brasil que utilizan, en algunas medidas, ese concepto. Fueron utilizados los descriptores "Ecodesenvolvimento" y 
"Brasil" para realizar la investigación de artículos en la base "Periódicos Capes", en el mes de junio del respectivo año de la investigación. Fueron identificados 54 artículos, de las cuales 24 fueron descartados por no cumplir con las especificidades de la investigación, o sea, se utilizó el concepto de "Ecodesenvolvimento" en investigación realizadas en Brasil. No se delimitó periodo temporal para esa búsqueda.

En principio fueron analizadas las palabras utilizadas en títulos de las publicaciones, de manera a identificar las frecuencias del uso de estas mismas palabras. Se denota que fueron identificadas las palabras con mayor "sustentabilidade", "sustentável", "ecodesenvolvimento" y "ambientais", en esa orden. De esta forma, se percibe que las investigaciones desarrolladas en Brasil, pueden relacionar el ecodesarrollo las cuestiones ambientales del país (Figura 1):

Figura 1 - Nubes de palabras de los títulos de los artículos

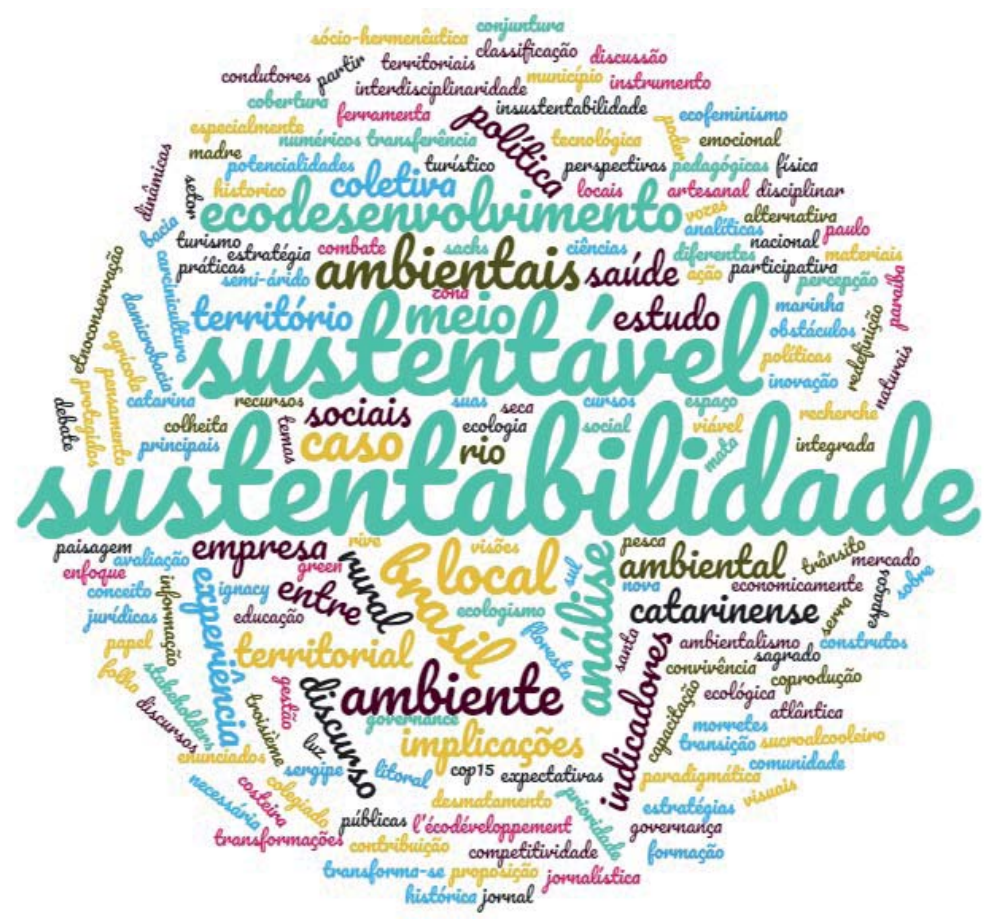

Fuente: Datos de la investigación, elaborado a partir de Word Clouds (2017). 
Secuencialmente se observa la frecuencia de palabras contenidas en las palabras-claves de las publicaciones. En estas nubes se puede desprender las palabras en destaques, o sea, sustentabilidade, sustentável, ambiente, meio, ambiental y local - en esta perspectiva de orden mayor a menor frecuencia. Aquellos en mayor destaque (sustentabilidade e sustentável), también fueron destaque en las nubes de palabras anterior. Se observó el uso de la palabra "local", término que relaciona con las descripciones de Sachs (1986) al considerar que el ecodesarrollo valoriza las características locales de los ecosistemas.

Figura 2 - Nubes de palabras de las palabras-clave de los artículos

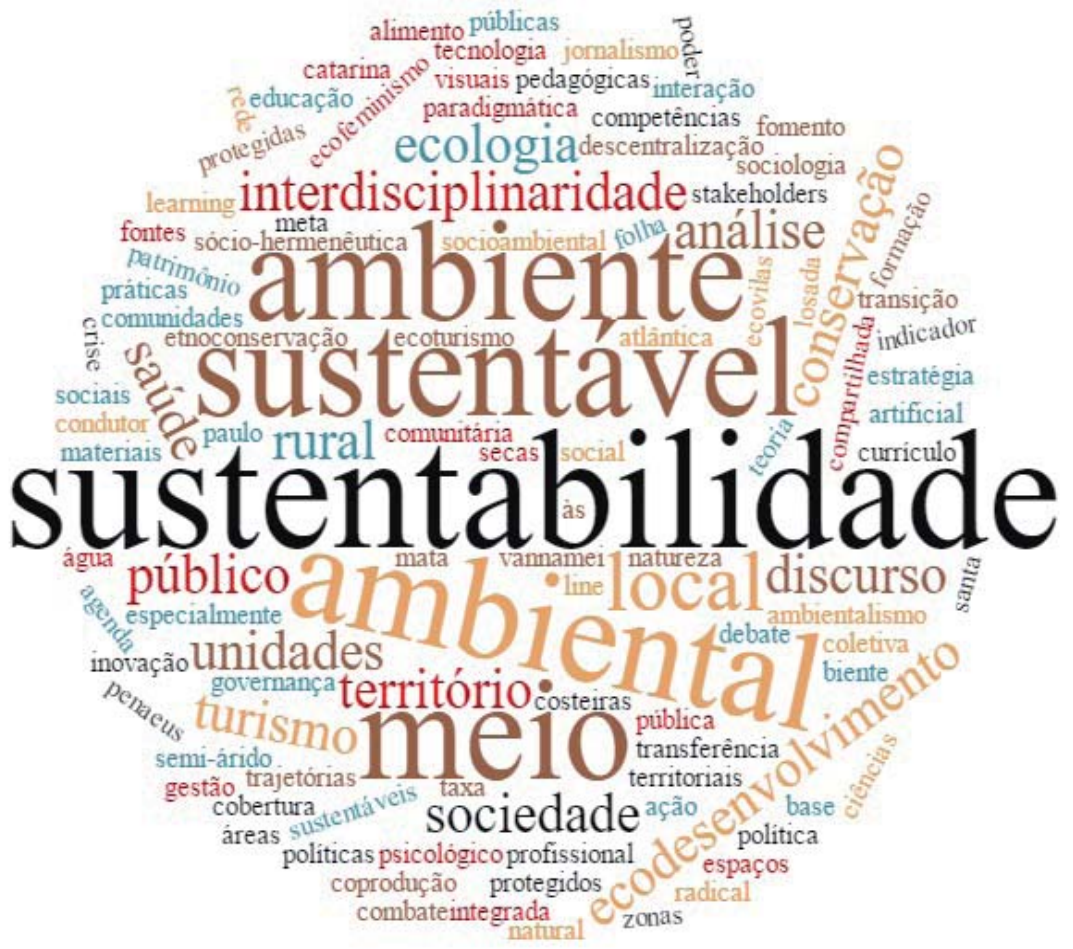

Fuente: Datos de investigación, elaborado a partir de Word Clouds (2017).

Se observó también el número de publicaciones realizadas por año y número total de autores y de coautores que realizaron estas publicaciones, sobre el asunto investigado (Figura 3). De esta forma, se identificó que en 
año del 2014 hubo el mayor número de publicaciones y en 2013 de autores y de coautores.

Figura 3 - Número de publicaciones, de autores y de coautores por año

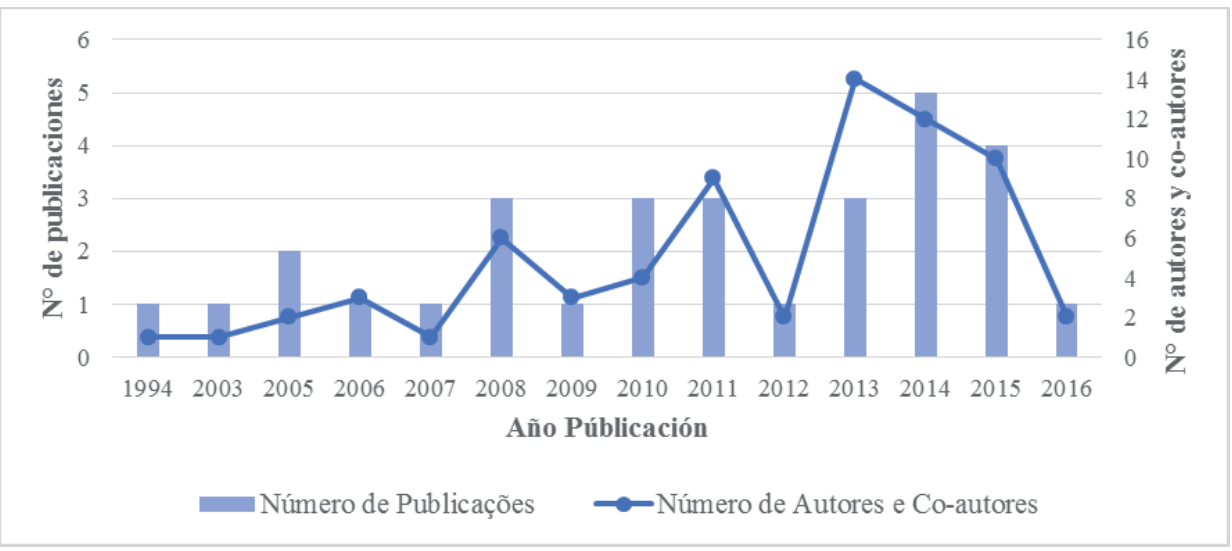

Fuente: Datos de la investigación (2017).

Fueron identificadas publicaciones en 18 revistas y considerados los Qualis Capes para el área de Ciencias Ambientales y Planeamiento Urbano y Regional/Demografía. La elección se debe la relación de las investigaciones con el área ambiental y considerando que el ecodesarrollo está relacionado al desarrollo. Apropiadamente 54\% de las revistas están clasificadas en el Qualis B1 para las Ciencias Ambientales y 28\% para el Planeamiento Urbano y Regional/Demografía. Además de eso, se percibe que, en la última área de evaluación, el número de revistas no poseen clasificación Qualis para ninguna de las áreas escogidas, 4 revistas para el área de Planeamiento Urbano y Regional/Demografía y 2 revistas para de Ciencias Ambientales. Por fin, se deprende que la revista Ambiental \& Sociedad es aquella con mayor número de poblaciones (8 artículos). 
Cuadro 1-Revistas en que fueron realizadas las publicaciones y Qualis Capes

\begin{tabular}{|l|c|c|}
\hline \multirow{2}{*}{\multicolumn{1}{|c|}{ Revistas }} & \multicolumn{2}{c|}{ Qualis } \\
\cline { 2 - 3 } & $\begin{array}{c}\text { Ciências } \\
\text { Ambientais }\end{array}$ & $\begin{array}{c}\text { Planejamento Urbano e } \\
\text { Regional/ Demografia }\end{array}$ \\
\hline Acta Scientiarum & B1 & B3 \\
\hline Ambiente \& Sociedade & A2 & A2 \\
\hline Ciência \& Saúde Coletiva & B1 & A2 \\
\hline Desenvolvimento e Meio Ambiente & B1 & B1 \\
\hline Estudos Feministas & B1 & A2 \\
\hline Holos & B4 & B1 \\
\hline Informação \& Sociedade: Estudos & - & - \\
\hline Política \& Sociedade & B2 & B1 \\
\hline Rac & B1 & B1 \\
\hline Revista de Administração Pública & B1 \\
\hline Revista Brasileira de Ciência do Solo & B1 & B2 \\
\hline Revista Brasileira de Pós-Graduação & B2 & B2 \\
\hline Revista da Faculdade de Bibliotecono- \\
mia e Comunicação da UFRGS & B2 & - \\
\hline Revista Econômica do Nordeste & - & - \\
\hline Revista Franco-Brasileira de Geografia & B2 & B1 \\
\hline Revista Nera & B3 & B2 \\
\hline Sociedade e Estado & B1 & - \\
\hline Turismo em Análise & B4 & B3 \\
\hline
\end{tabular}

Fuente: Datos de la investigación (2017).

En relación la localización de las publicaciones de los artículos, se percibe que fueron contemplados 9 estados y el Distrito Federal para publicación, con todo existe concentración de 43\% de las publicaciones en el São Paulo (Figura 4) dos publicaciones. 
Figura 4 - Localización de las publicaciones sobre ecodesarrollo en Brasil

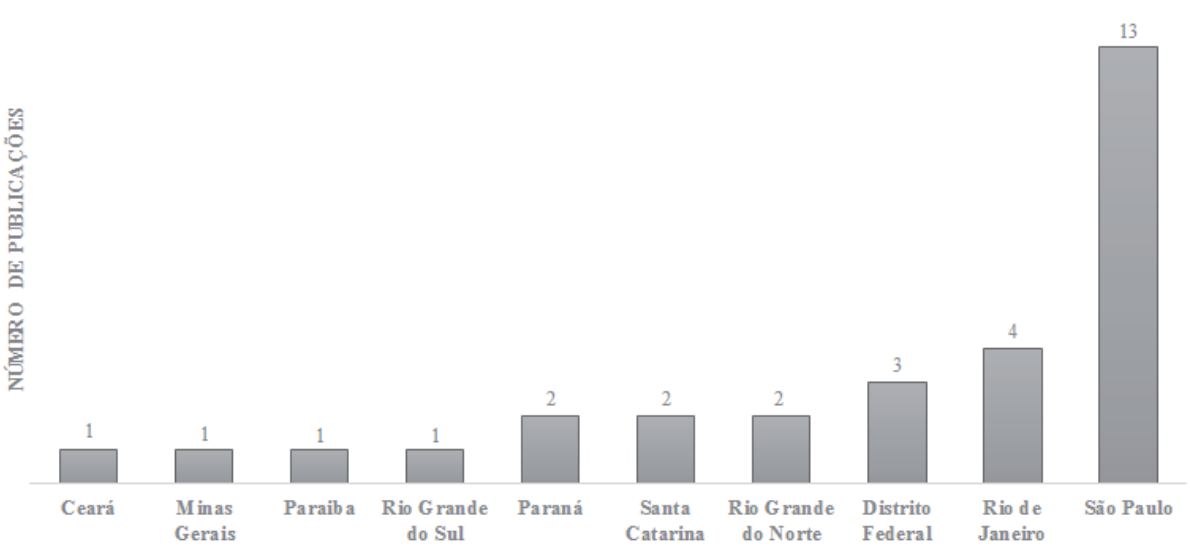

Fuente: Datos de la winvestigación (2017).

También fueron consultadas el número de citación de los respectivos artículos en el Google Académico. El Cuadro 2 índica las citaciones identificadas, se puede afirmar que fueron diversificadas. El mayor número de citaciones es de 196 y relacionada a la sostenibilidad con la educación. Cabe registrar también que nueve artículos todavía no fueron citados por otros autores. Esas publicaciones no referenciadas fueron publicadas en los últimos cinco años, en la mayoría de las veces en los últimos tres años. Puede ser el motivo por la falta de citaciones.

Cuadro 2 - Número de citaciones por artículo

\begin{tabular}{|l|c|c|}
\hline \multicolumn{1}{|c|}{ Título del Artículo } & $\begin{array}{c}\text { Número de } \\
\text { Citaciones }\end{array}$ & $\begin{array}{c}\text { Autor(es) } \\
\text { (ano) }\end{array}$ \\
\hline $\begin{array}{l}\text { O discurso da sustentabilidade e suas implicações } \\
\text { para a educação }\end{array}$ & 196 & Lima (2003) \\
\hline $\begin{array}{l}\text { Entre o combate à seca e a convivência com o } \\
\text { Semi-Árido: políticas públicas e transição paradig- } \\
\text { mática }\end{array}$ & 121 & $\begin{array}{c}\text { Alves da Silva } \\
\text { (2007) }\end{array}$ \\
\hline $\begin{array}{l}\text { Ecologismo, ambientalismo e ecologia política: dife- } \\
\text { rentes visões da sustentabilidade e do território }\end{array}$ & 47 & $\begin{array}{c}\text { Jatobá, Cidade } \\
\text { e Vargas } \\
\text { (2009) }\end{array}$ \\
\hline $\begin{array}{l}\text { Ciências sociais e ambiente rural: principais temas } \\
\text { e perspectivas analíticas }\end{array}$ & 41 & $\begin{array}{c}\text { Brandenburg } \\
\text { (2005) }\end{array}$ \\
\hline
\end{tabular}


Ecodesarrollo como estrategia para la sostenibilidad: un análisis socioambiental basado en la obra de Ignacy Sachs

\begin{tabular}{|c|c|c|}
\hline Título del Artículo & $\begin{array}{l}\text { Número de } \\
\text { Citaciones }\end{array}$ & $\begin{array}{l}\text { Autor(es) } \\
\text { (ano) }\end{array}$ \\
\hline $\begin{array}{l}\text { Gestão integrada e participativa da pesca arte- } \\
\text { sanal: potencialidades e obstáculos no litoral do } \\
\text { Estado de Santa Catarina }\end{array}$ & 36 & $\begin{array}{c}\text { Rebouças, } \\
\text { Filardi e Vieira } \\
\text { (2006) } \\
\end{array}$ \\
\hline $\begin{array}{l}\text { Por um pensamento ambiental histórico: O caso } \\
\text { do Brasil }\end{array}$ & 34 & Duarte (2005) \\
\hline $\begin{array}{l}\text { Espaços territoriais especialmente protegidos: } \\
\text { conceito e implicações jurídicas }\end{array}$ & 33 & $\begin{array}{c}\text { Pereira e } \\
\text { Scardua } \\
(2008)\end{array}$ \\
\hline $\begin{array}{l}\text { Espaço emocional e indicadores de sustentabili- } \\
\text { dade }\end{array}$ & 23 & $\begin{array}{l}\text { Paulista, } \\
\text { Varakis e } \\
\text { Montibeller- } \\
\text { Filho (2008) }\end{array}$ \\
\hline $\begin{array}{l}\text { Transferência tecnológica e inovação por meio da } \\
\text { sustentabilidade }\end{array}$ & 21 & $\begin{array}{l}\text { Freitas et al. } \\
(2012)\end{array}$ \\
\hline Informação ambiental- uma prioridade nacional? & 15 & Targino (1994) \\
\hline $\begin{array}{l}\text { E a política agrícola transforma-se em instrumento } \\
\text { do desenvolvimento sustentável }\end{array}$ & 8 & Mello (2008) \\
\hline $\begin{array}{l}\text { Ação coletiva e coprodução para o desenvolvi- } \\
\text { mento rural: um estudo de caso do Colegiado de } \\
\text { Desenvolvimento Territorial da Serra Catarinense }\end{array}$ & 6 & \begin{tabular}{|c|} 
Ribeiro, \\
Andion e \\
Burigo (2015) \\
\end{tabular} \\
\hline $\begin{array}{l}\text { Saúde, meio ambiente e território: uma discussão } \\
\text { necessária na formação em saúde }\end{array}$ & 6 & $\begin{array}{l}\text { Souza e } \\
\text { Andrade } \\
(2014)\end{array}$ \\
\hline $\begin{array}{l}\text { De Ignacy Sachs, La troisième rive: à la recherche } \\
\text { de l'écodéveloppement }\end{array}$ & 4 & Gentil (2011) \\
\hline $\begin{array}{l}\text { O Papel de Condutores Ambientais Locais e de } \\
\text { Cursos de Capacitação no Ecodesenvolvimento } \\
\text { Turístico e as Expectativas Sociais no Sul do Brasil }\end{array}$ & 4 & $\begin{array}{l}\text { Ribas e } \\
\text { Hickenbick } \\
\text { (2012) }\end{array}$ \\
\hline $\begin{array}{l}\text { Análise sócio-hermenêutica do Discurso da Sus- } \\
\text { tentabilidade a Partir de Materiais Visuais }\end{array}$ & 2 & $\begin{array}{c}\text { Coelho, Godoi } \\
\text { e Coelho } \\
(2015)\end{array}$ \\
\hline $\begin{array}{l}\text { Discursos e vozes na cobertura jornalística das } \\
\text { COP15 e } 16\end{array}$ & 2 & $\begin{array}{l}\text { Girardi et al. } \\
(2013)\end{array}$ \\
\hline $\begin{array}{l}\text { Etnoconservação de recursos naturais na zona } \\
\text { costeira catarinense: uma análise das transforma- } \\
\text { ções da paisagem na bacia do Rio da Madre, à luz } \\
\text { do enfoque de ecodesenvolvimento }\end{array}$ & 2 & $\begin{array}{l}\text { Prudencio, } \\
\text { Vieira e } \\
\text { Fonseca } \\
\text { (2014) }\end{array}$ \\
\hline
\end{tabular}




\begin{tabular}{|c|c|c|}
\hline Título del Artículo & $\begin{array}{l}\text { Número de } \\
\text { Citaciones }\end{array}$ & $\begin{array}{l}\text { Autor(es) } \\
\text { (ano) }\end{array}$ \\
\hline $\begin{array}{l}\text { Green governance: a proposição de construtos de } \\
\text { governança coletiva para o desenvolvimento sus- } \\
\text { tentável local }\end{array}$ & 2 & $\begin{array}{l}\text { Padilha e } \\
\text { Verschoore } \\
(2013)\end{array}$ \\
\hline Ecofeminismo e comunidade sustentável & 1 & $\begin{array}{l}\text { Flores e } \\
\text { Trevisan } \\
\text { (2015) } \\
\end{array}$ \\
\hline $\begin{array}{l}\text { Indicadores numéricos como ferramenta para ava- } \\
\text { liação da sustentabilidade ecológica da carcinicul- } \\
\text { tura marinha em Sergipe, Brasil }\end{array}$ & 1 & $\begin{array}{l}\text { Muhlert et al. } \\
\text { (2013) }\end{array}$ \\
\hline $\begin{array}{l}\text { A classificação disciplinar no mercado dos enun- } \\
\text { ciados ambientais }\end{array}$ & 0 & $\begin{array}{l}\text { Martins } \\
(2015)\end{array}$ \\
\hline $\begin{array}{l}\text { A colheita da floresta: Uma estratégia de desen- } \\
\text { volvimento social e economicamente viável ao } \\
\text { desmatamento? }\end{array}$ & 0 & Oliveira (2010) \\
\hline A insustentabilidade em trânsito & 0 & $\begin{array}{l}\text { Lindoso } \\
(2010)\end{array}$ \\
\hline $\begin{array}{l}\text { Desenvolvimento sustentável, redefinição do po- } \\
\text { der local e turismo como alternativa de desenvol- } \\
\text { vimento local }\end{array}$ & 0 & $\begin{array}{c}\text { França e } \\
\text { Araujo (2010) }\end{array}$ \\
\hline $\begin{array}{l}\text { Desenvolvimento territorial sustentável: uma nova } \\
\text { experiência na Mata Atlântica }\end{array}$ & 0 & $\begin{array}{l}\text { Tiepolo e } \\
\text { Denardin } \\
(2016)\end{array}$ \\
\hline $\begin{array}{l}\text { Dinâmicas física e histórica na conjuntura do de- } \\
\text { senvolvimento sustentável }\end{array}$ & 0 & $\begin{array}{l}\text { Teodoro } \\
(2014)\end{array}$ \\
\hline $\begin{array}{l}\text { Interdisciplinaridade e práticas pedagógicas no } \\
\text { Ecodesenvolvimento: análise da experiência da } \\
\text { microbacia do Rio Sagrado, Morretes, PR }\end{array}$ & 0 & $\begin{array}{l}\text { Grimm et al. } \\
\qquad(2015)\end{array}$ \\
\hline $\begin{array}{l}\text { O debate sobre meio ambiente no jornal Folha de } \\
\text { São Paulo entre } 1992 \text { e } 2008\end{array}$ & 0 & $\begin{array}{l}\text { Cervi e } \\
\text { Massuchin } \\
(2011)\end{array}$ \\
\hline $\begin{array}{l}\text { Percepção de stakeholders acerca da contribuição } \\
\text { das estratégias ambientais para a sustentabilidade } \\
\text { do município e competitividade da empresa: estu- } \\
\text { do de caso em empresa do setor sucroalcooleiro } \\
\text { no estado da Paraíba }\end{array}$ & 0 & $\begin{array}{l}\text { Barbosa, } \\
\text { Cândido } \\
\text { e Barbosa } \\
\text { (2014) }\end{array}$ \\
\hline
\end{tabular}

Fuente: Datos de la investigación (2017). 
Lima (2003), autor del trabajo con mayor número de citaciones, considera que Ignacy Sachs, con el concepto de ecodesarrollo, y la Comisión de Brundtland posibilitaron que las discusiones sobre desarrollo sostenible se tornaron globales. Con el tiempo fue posible la creación de nueva estrategia de desarrollo, ahora pautada en la sostenibilidad de los elementos: económico, social y ambiental.

\section{CONSIDERACIONES FINALES}

Al conceptuar el ecodesarrollo, Sachs intenta concienciar a la sociedad sobre las posibles catástrofes naturales que pueda acontecer sobre el planeta en la hipótesis del mantenimiento del sistema de producción sin preocupación ecológica. Los efectos más serios de la utilización indiscriminada de los recursos naturales, o sea, sin prerrequisitos de conservación de la biodiversidad, pueden ser: lluvia ácida, calentamiento de la tierra, reducción de capa de ozono, desertificación generalizada y extinción de las especies. Los efectos de los peligros mencionados se pueden no sentir en la actualidad, pero sí, en un futuro venidero. Así, el autor trae para la sociedad una nueva perspectiva sobre la manera de cómo se debería realizar la extracción y uso de los recursos naturales sin causar ninguna perturbación al ecosistema, creando un equilibrio entre las actividades humanas y el medio ambiente.

Otro problema registrado es el estilo de producción capitalista presente en la mayoría de las naciones, lo que puede ser clasificado como desarrollo excluyente y concentrador de riqueza. Ese estilo de desarrollo es responsable por la existencia de las desigualdades sociales en las naciones, pues el que importa es la internacionalización de lucros y la externalización de los costos sociales, impactando a la sociedad y el medio ambiente. Así, al emplearse los procesos de ecodesarrollo, se cree en acciones para la existencia de crecimiento económico sin destrucción del medio ambiente, creando balance adecuado entre las acciones del hombre en la búsqueda de riqueza, la conservación del medio ambiente y divide los dividendos entre las diferentes camadas sociales de forma más igualitaria, esto es, la introducción de estilo de desarrollo incluyente, o sea, que elimina o reduce las desigualdades sociales.

La pobreza es responsable por todo desequilibrio ambiental, y esa 
cuestión se torna bien severa en países del Tercer Mundo, países que dependen de las economías de otras naciones. En países donde la pobreza es endémica, allí las poblaciones estarán sujetas a catástrofes ecológicas o de otras naturalezas. Eso quiere decir que, en países pertenecientes al Tercer Mundo, en ellos la exploración inadecuada de los recursos naturales tiende a ser realizada de forma deliberada y excesiva a partir de tecnologías obsoletas y que no respetan las normas ambientales. Además de eso, esas tecnologías pueden tener fuerte capacidad destructiva sobre el medio ambiente, tornando así la actividad de extracción en poco tiempo económicamente no viable y socialmente injusta. En el largo plazo, esa forma de extracción de los recursos naturales con finalidad productiva puede impactar el medio ambiente, reduciendo los recursos y los servicios con finalidad productiva.

Existe, en las obras de Sachs, una integración entre el aparato tecnológico y social. En la percepción del autor, es necesario que la tecnología usada en el proceso de transformación de los recursos naturales en bienes y servicios consumibles sea limpias y capaz de mantener un equilibrio ecológico y garantir su sostenibilidad con el tiempo. Así, la tecnología puede ser entendida como aspecto crucial para dinamizar y estructurar la organización social, mejorando de esa calidad de vida de la sociedad. Esa nueva tecnología, por tanto, no es excluyente, pues ella auxilia en la permanencia de los individuos en su espacio rural. Ella debe llegar a todos aquellos que desarrollan prácticas que utilicen recursos naturales. De esa forma, la innovación debe ser eficiente y con costos bajos para tornarse accesible.

\section{REFERENCIAS}

ALVES DA SILVA, R. M. Entre o combate à seca e a convivência com o semi-árido: políticas públicas e transição paradigmática. Revista Econômica do Nordeste, Fortaleza, v. 38, n. 3, p. 466-85, 2007.

ANDRADE, D. C. Economia e meio ambiente: aspectos teóricos e metodológicos nas visões neoclássica e da economia ecológica. Leituras de Economia Política, Campinas, v. 14, p. 1-31, 2008.

ARAÚJO, K. K. S.; BARROSO, C. M. R.; SOUZA, E. J. C. Ecodesenvolvimento e 
Ecodesarrollo como estrategia para la sostenibilidad: un análisis socioambiental basado en la obra de Ignacy Sachs

desenvolvimento sustentável: conceitos e divergências. Revista Reflexões e Práticas Geográficas, Maceió, v. 1, n. 1, p. 45-57, 2014.

BARBOSA, M. F. N., CANDIDO, G. A.; BARBOSA, E. M. Percepção de stakeholders acerca da contribuição das estratégias ambientais para a sustentabilidade do município e competitividade da empresa: estudo de caso em empresa do setor sucroalcooleiro no estado da Paraíba. Holos, Natal, v. 1, p. 191-209, 2014.

BRANDENBURG, A. Ciências sociais e ambiente rural: principais temas e perspectivas analíticas. Ambiente \& Sociedade, São Paulo, v. 8, n. 1, p. 1-13, 2005.

CERVI, E. U.; MASSUCHIN, M. G. O debate sobre meio ambiente no jornal Folha de São Paulo entre 1992 e 2008. Política \& Sociedade, v. 10, n. 19, p. 239-73, 2011.

COMISSÃO MUNDIAL SOBRE O MEIO AMBIENTE E DESENVOLVIMENTO (CMMAD). Nosso futuro comum. Rio de Janeiro: Editora da FGV, 1988.

COELHO, A. L. A. L.; GODOI, C. K.; COELHO, C. Análise sócio-hermenêutica do discurso da sustentabilidade a partir de materiais visuais. RAC, Rio de Janeiro, $\mathrm{v}$. 19, n. 5, p. 649-70, 2015.

DUARTE, R. H. Por um pensamento ambiental histórico: o caso do Brasil. LuzoBrazilian Review, v. 41, n. 2, p. 144-61, 2005.

FERNANDEZ, B. P. M. Ecodesenvolvimento, desenvolvimento sustentável e economia ecológica: em que sentido representam alternativas ao paradigma de desenvolvimento tradicional? Desenvolvimento e Meio Ambiente, v. 23, p. 109-20, 2011.

Flores, B. N.; Trevisan, S. P. Ecofeminismo e comunidade sustentável. Revista Estudos Feministas, Florianópolis, v. 23, n. 1, p. 11-34, 2015.

FRANÇA, J. P.; ARAUJO, I. J. Desenvolvimento sustentável, redefinição do poder local e turismo como alternativa de desenvolvimento local. Holos, v. 2, p. 60-70, 2010.

FREITAS, C. S. G.; MAÇANEIRO, M. B.; KUHL, M. R.; SEGATTO, A. P.; OLIVEIRA, S. L. D.; LIMA, L. F. Transferência tecnológica e inovação por meio da sustentabilidade. Revista de Administração Pública, Rio de Janeiro, v. 46, n. 2, p. 363-84, 2012.

GENTIL, V. De Ignacy Sachs, la troisième rive: à la recherche de l'écodéveloppement. A terceira margem: à procura do ecodesenvolvimento. Sociedade e Estado, Brasília, DF, v. 26, n. 1, p. 269-74, 2011.

GIRARDI I. M. D.; MASSIERER, C.; MORAES, C. H.; LOOSE, E. B.; NEULS, G.; SCHWAAB, R.; CAMANA, A.; GERTZ, L. Discursos e vozes na cobertura jornalística das COP15 e 16. Em Questão, Porto Alegre, v. 19, n. 2, p. 176-94, 2013.

GRIMM, I. J.; DIAS, A.; SAMPAIO, C. A. C.; FERNANDES, V. Interdisciplinaridade e práticas pedagógicas no ecodesenvolvimento: análise da experiência da microbacia do Rio Sagrado, Morretes, PR. Ambiente \& Sociedade, São Paulo, v. 18, n. 1, p. 
Luciana Virginia Mario BERNARDO; Cesar Benites Mario ZIDORA; Maycon Jorge Ulisses Saraiva FARINHA; Nelson David Lesmo DUARTE

115-32, 2015.

JATOBÁ, S. U. S.; CIDADE, L. C. F.; VARGAS, G. M. Ecologismo, ambientalismo e ecologia política: diferentes visões da sustentabilidade e do território. Sociedade e Estado, Brasília-DF, v. 24, n. 1, p. 47-87, 2009.

LIMA, G. C. O discurso da sustentabilidade e suas implicações para a educação. Ambiente \& Sociedade, São Paulo, v. 6, p. 99-119, 2003.

LINDOSO, D. P. A insustentabilidade em trânsito. Ambiente \& Sociedade, São Paulo, v. 14, n. 2, p. 433-5, 2010.

MARTINS, R. C. A classificação disciplinar no mercado dos enunciados ambientais. Revista Brasileira de Ciências Sociais, São Paulo, v. 30, n. 87, p. 97-114, 2015.

MCCORMICK, J. Rumo ao paraíso: a história do movimento ambientalista. Traducción Marco Antonio Esteves da Rocha e Renato Aguiar. Rio de Janeiro: Relume-Dumará, 1992.

MEADOWS, D. H.; MEADOWS, D. L.; RANDERS, J.; BEHRENS III, W. Limits to growth. Nova York: Universe Books. 1972.

MELLO, N. A. E a política agrícola transforma-se em instrumento do desenvolvimento sustentável. Revista NERA, Presidente Prudente, v. 11, n. 2, p. 68-85, 2008.

MONTIBELLER FILHO, G. O mito do desenvolvimento sustentável: meio ambiente e custos sociais no moderno sistema de produtor de mercadorias. 2. ed. Florianópolis, SC: Editora da UFSC, 2004.

MUHLERT, A. C. S.; LIMA, J. S. G.; MACHADO, L.; EVANGELISTA, R. A. Indicadores numéricos como ferramenta para avaliação da sustentabilidade ecológica da carcinicultura marinha em Sergipe, Brasil. Interciência, Santiago, Chile, v. 38, n. 8, p. 615-20, ago. 2013.

OLIVEIRA, D. F.; MONTEIRO, L. V. G. Ecodesenvolvimento: uma abordagem sob o contributo de Ignacy Sachs. Revista de Direito, Economia e Desenvolvimento Sustentável, Florianópolis, v. 1, n. 2, p. 29-48, 2015.

OLIVEIRA, P. A. A colheita da floresta: uma estratégia de desenvolvimento social e economicamente viável ao desmatamento? Confins - Revista Franco-Brasileira de Geografia, Paris, n. 9, 2010.

PADILHA, L. G. O.; VERSCHOORE, J. R. S. Green governance: a proposição de construtos de governança coletiva para o desenvolvimento sustentável local. Ambiente \& Sociedade, São Paulo, v. 16, n. 2, p. 153-72, 2013.

PAULISTA, G.; VARVAKIS, G.; MONTIBELLER-FILHO, G. Espaço emocional e indicadores de sustentabilidade. Ambiente \& Sociedade, São Paulo, v. 11, n. 1, p. 185-200, 2008. PEREIRA, P. F.; SCARDUA, F. P. Espaços territoriais especialmente protegidos: 
Ecodesarrollo como estrategia para la sostenibilidad: un análisis socioambiental basado en la obra de Ignacy Sachs

conceito e implicações jurídicas. Ambiente \& Sociedade, São Paulo, v. 11, n. 1, p. 81-97, 2008.

PRUDENCIO, J. M.; VIEIRA, P. F.; FONSECA, A. L. O. Etnoconservação de recursos naturais na zona costeira catarinense: uma análise das transformações da paisagem na bacia do Rio da Madre, à luz do enfoque de ecodesenvolvimento. Desenvolvimento e Meio Ambiente, n. 32, p. 41-60, 2014.

RAYNAUT, C.; ZANONI, M. La construction de l'interdiciplinarité en formation integrée de l'environnement et du développement. Paris: Unesco (Document préparé pour la Réunion sur les Modalités de travail de Chaires Unesco du Développment Durable. Curitiba, 1- 4 juillát 93- mimeo). 1993.

REBOUÇAS, G. N.; FILARDI, A. C. L.; VIEIRA, P. F. Gestão integrada e participativa da pesca artesanal: potencialidades e obstáculos no litoral do Estado de Santa Catarina. Ambiente \& Sociedade, São Paulo, v. 9, n. 2, p. 83-104, 2006.

RIBAS, L. C. C.; HICKENBICK, C. O papel de condutores ambientais locais e de cursos de capacitação no ecodesenvolvimento turístico e as expectativas sociais no Sul do Brasil. Turismo em Análise, São Paulo, v. 23, n. 1, p. 143-65, 2012.

RIBEIRO, A. C.; ANDION, C.; BURIGO, F. Ação coletiva e coprodução para o desenvolvimento rural: um estudo de caso do Colegiado de Desenvolvimento Territorial da Serra Catarinense. Revista de Administração Pública, Rio de Janeiro, v. 49, n. 1, p. 119-40, 2015.

SACHS, I. Stratégies de l'écodéveloppement. Paris: Les Editions Ouvrières, 1980. 140p. . Ecodesenvolvimento crescer sem destruir. Terra dos Homens. 1. ed. São Paulo: Editora Vértice, 1986. 207p.

. Recursos, emprego e financiamento do desenvolvimento: produzir sem destruir- O caso do Brasil. Revista de Economia Política, v. 10, n. 1, p. 111-32, 1990.

. Transition strategies for the 21st. century. Nature and Resources, v. 28, n. 1, 1993.

. Brasil rural: da redescoberta à invenção. Estudos Avançados, São Paulo, v. 15, n. 43, p. 75-82, 2001.

2002.

. Caminhos para o desenvolvimento sustentável. Rio de Janeiro: Garamond,

. A terceira margem: em busca do ecodesenvolvimento. São Paulo: Companhia das Letras, 2009.

. Barricadas de ontem, campos de futuro. Estudos Avançados, São Paulo, v. 24, n. $68,2010$.

SALAMONI, G.; Gerardi, L. H. O. Princípios sobre o ecodesenvolvimento e suas 
Luciana Virginia Mario BERNARDO; Cesar Benites Mario ZIDORA; Maycon Jorge Ulisses Saraiva FARINHA; Nelson David Lesmo DUARTE

relações com a agricultura familiar. In: GERARDI, L. H. O.; MENDES, I. A. (Org.). Teoria, técnicas, espaços e atividades - temas da Geografia Contemporânea. Rio Claro, SP: AGETEO, p. 73-96, 2001

SOLOW, R. M. The economics of resources or the resources of economics. The American Economic Review, v. 64, n. 2, p. 1-14, 1974.

SOUTH COMMISSION. The challenge to the South. Oxford: Oxford University Press, 1990.

SOUZA, C. L.; ANDRADE, C. S. Saúde, meio ambiente e território: uma discussão necessária na formação em saúde. Ciência \& Saúde Coletiva, Rio de Janeiro, v. 19, n. 10, 4113-22, 2014.

TARGINO, M. G. Informação ambiental- uma prioridade nacional? Informação \& Sociedade: Estudos, João Pessoa, v. 4, n. 1, p. 38-61, 1994.

TEODORO, P. H. M. Dinâmicas física e histórica na conjuntura do desenvolvimento sustentável. Acta Scientiarum, Maringá, v. 36, n. 1, p. 73-84, 2014.

TIEPOLO, L. M.; DENARDIN, V. F. Desenvolvimento territorial sustentável: uma nova experiência na Mata Atlântica. Revista Brasileira de Pós-Graduação, v. 13, n. 32, p. 865-88, 2016. 